\title{
Gestão da informação em organizações virtuais: uma nova questão para a coordenação interorganizacional no setor público
}

Henrique Silveira

Doutorando em ciência da informação pela Universidade de Brasília; Mestre em ciência da informação pela Universidade de Brasília; Analista do Banco Central do Brasil

E-mail: henriquefrs@yahoo.com.br

\section{Resumo}

O artigo destaca a importância da reflexão sobre as implicações e possibilidades que os conceitos relativos a organizações virtuais podem trazer para a coordenação interorganizacional no âmbito do planejamento governamental, de forma a contribuir para incrementar a efetividade da ação de governo, por meio de um ambiente informacional cooperativo. Aspectos como gestão cooperativa da informação, cultura organizacional, poder e controle, fronteiras e estruturas organizacionais e confiança deverão ser observados no contexto do planejamento governamental, caso se pretenda evoluir na aplicação do conceito de organizações virtuais ao setor público.

\section{Palavras-chave}

Planejamento governamental; Coordenação interorganizacional; Gestão da informação.

Information management in virtual organizations: a new issue to public sector inter-organizational coordination

\begin{abstract}
The article highlights the importance of a reflection about the impacts and possibilities that the concepts related to virtual organisations may bring to the interorganisational coordination in the governmental planning. Such interorganisational coordination may contribute to improve the effectiveness of the governmental action, by means of a cooperative informational environment. Aspects like, cooperative information management, organisational culture, power and control, boundaries and organizational structures, as well as trust must be object of attention, in case one wants to evolve towards applying the concept of virtual organizations to the public sector.
\end{abstract}

\section{Keywords}

Governmental planning; Interoganisational coordination; Information management.

\section{INTRODUÇÃO}

É crescente o envolvimento de organizações públicas e privadas com alianças e parcerias, conforme estudos a serem apresentados neste trabalho. No novo cenário colaborativo, uma nova abordagem para o planejamento das organizações precisa ser desenvolvida, com especial atenção para o setor público, em cujo ambiente a escassez de recursos pode ser total ou parcialmente suprida pela criação ou incremento de redes entre organizações e sistemas.

Essa nova abordagem para a coordenação interorganizacional no âmbito do planejamento governamental pode resultar em algum tipo de "organização virtual" para o setor público, à semelhança do que já ocorre no setor privado, requerendo o suporte de sistemas interorganizacionais, habilidades específicas e compromisso por parte das organizações envolvidas.

Para explorar o tema, o trabalho foi estruturado em sete tópicos, incluindo a introdução. Na seqüência, é apresentado o contexto que condiciona e estimula as organizações a migrar para a nova abordagem de colaboração. Em seguida, são apresentados os conceitos de "ambiente informacional cooperativo" e de "organização virtual". Os dois tópicos seguintes abordam a questão da gestão da informação no âmbito da coordenação interorganizacional, tanto no processo de planejamento propriamente dito, quanto na fase de implementação dos planos.

A conclusão destaca a importância da reflexão sobre as implicações e possibilidades que os conceitos relativos a organizações virtuais podem trazer para a gestão da informação no âmbito da coordenação interorganizacional no setor público, de forma a contribuir para incrementar a efetividade da ação de governo.

\section{CONTEXTO}

É crescente o envolvimento das organizações com alianças e parcerias que as levam a agir além das tradicionais fronteiras organizacionais (AUSTIN, 2002; BRADNER, 2002; GLENDINNING, 2003; MOLINA, 2001). Os desafios que as organizações enfrentam

Ci. Inf., Brasília, v. 34, n. 2, p. 70-80, maio/ago. 2005 
requerem cada vez mais o esforço colaborativo e a efetiva ação coordenada de várias organizações, incluindo governo, setor privado e terceiro setor. Colaborar tornouse um imperativo.

Nesta nova era, tradicionais conceitos são abandonados ou questionados, e o próprio conceito de "organização" está mudando, de forma a refletir os desafios inerentes ao novo ambiente. Na sociedade interconectada, a fonte primária de criação de valor mudou a ênfase da produtividade para os relacionamentos, e a capacidade de colaborar precisa se tornar uma competência-chave para a organização (CASTELLS, 1998; FILOS, 2001). Molina (2001) também afirma que a mais importante competência é a habilidade para integrar as competências dos parceiros.

Também para Bradner (2002), tecnologias "virtuais" e globalização estão conduzindo a um blurring (perda de nitidez) das fronteiras organizacionais. Burnett (2000) afirma que as pessoas tendem a manter a atenção em recursos úteis e que estão fora das fronteiras de suas organizações, e, atualmente, a tecnologia oferece muitas possibilidades de incorporação desses recursos à ação organizacional.

Riempp (1998) declara que as fronteiras organizacionais estão se dissolvendo, de forma a atingir uma reação mais rápida às necessidades do consumidor. Entretanto, quando as fronteiras organizacionais se tornam indistintas, por meio do compartilhamento de processos, expertise, pessoal e outros recursos, novas fontes de integridade organizacional, identidade e propriedade do capital intelectual precisam ser discutidas (MERALI, 2002).

Novas formas organizacionais são possíveis porque a tecnologia da informação tem a capacidade de mudar a configuração tradicional de espaço-tempo (SCHULTZE, 2000). Para Schultze, grandes transformações estão ocorrendo nas estruturas sociais e organizacionais, associadas com o uso intensivo das tecnologias de informação.

Acima de tudo, arranjos organizacionais mais flexíveis demandam e facilitam maneiras de pensar que transcendem estreitos domínios profissionais, de serviço ou de setor (GLENDINNING, 2003). Considerando que muitas habilidades e recursos essenciais para a organização estão fora de suas fronteiras, e, portanto, fora do controle direto da gerência, parcerias e alianças não devem mais ser vistas como opções, mas como uma necessidade. Para Filos (2001), sinergias são inerentes em relacionamentos interativos e multifacetados, e nenhuma organização hoje pode permanecer como "uma ilha”.

Nesse contexto, o desenvolvimento de ambientes de informação cooperativos é uma questão que tem se tornado mais e mais importante. Arcieri (2002) credita esse fato a uma explosão da disponibilidade de redes e ao contínuo crescimento da presença de sistemas de informação no dia-a-dia das organizações.

Entretanto, as tradicionais metodologias de desenvolvimento de sistemas de informação têm foco em necessidades informacionais específicas de uma organização (ALBERTIN, 1999; BIO, 1996; EINDOR; SEGEV, 1986; SAVIANI, 1998; SHIMIZU, 1994; YOURDON, 1990). Assim, as organizações têm desenvolvido seus próprios sistemas de informação sem necessariamente pensar em termos de cooperação com outras entidades fora de suas fronteiras. Agora que a conectividade é tecnicamente possível e economicamente viável, a integração entre sistemas legados se prova mais difícil de obter (ARCIERI, 2002).

Ademais, a migração para um ambiente colaborativo com a prevalência de relacionamentos interorganizacionais pode ser considerada uma mudança de paradigma, na forma sugerida por Kuhn (1982). Relacionamentos são, indubitavelmente, uma poderosa fonte de criação de valor, mas Spinosa (2001) afirma que é necessária a adoção de políticas que assegurem a geração, o uso, a manutenção e a disseminação do capital intelectual na rede, isto é, a gestão da informação em uma organização virtual, configurando o que se pode chamar de "ambientes informacionais cooperativos", objeto de atenção do próximo tópico.

\section{AMBIENTES INFORMACIONAIS COOPERATIVOS}

Em relação ao desenvolvimento de ambientes informacionais cooperativos, os problemas realmente difíceis quase nunca são os problemas técnicos, mas aqueles que têm uma natureza organizacional (ARCIERI, 2002). De fato, nos últimos anos, a disponibilidade de poder computacional e de conectividade a preços acessíveis tem alcançado níveis que seriam inacreditáveis apenas 20 anos atrás. Isso torna factível a implementação de quase qualquer solução técnica que seja necessária.

Para além de questões técnicas, o grande desafio é construir um sistema de informação cooperativo que seja capaz de dar suporte à maneira pela qual as organizações interagem, sendo minimamente intrusivo e favorecendo real e efetiva cooperação. A questão é a capacidade para integrar diferentes tipos de abordagens, arquiteturas, 
tecnologias e, acima de tudo, o entendimento sobre cada domínio em particular (SILVA, 2001).

Novas formas de interoperabilidade precisam ser alcançadas, e um dos objetivos mais difíceis de alcançar em um desenvolvimento de sistemas de informação cooperativos é a coerência dos conjuntos de dados, geralmente distribuídos. Para Arcieri (2002), por um lado, de fato, dados são independente e autonomamente gerenciados pelas várias organizações. Por outro lado, dados são necessários e são usados também fora da organização que os produz, gerencia e controla as mudanças.

Mutschke (2001) argumenta que a estrutura social é um fator-chave no entendimento da difusão de idéias, da transferência de conhecimento, e, mais importante, na criação de novo conhecimento. Também para Castelfranchi (2002) e Burnett (2000), o acesso à informação não é mais concebido apenas como um problema técnico, mas como um processo social, por causa de aspectos dominantes, como direitos, privacidade, papéis etc.

Assim, o real desafio na construção e gestão de ambientes informacionais cooperativos é o compartilhamento de significados, uma vez que a informação - como o conteúdo da interação social - provê os materiais brutos a partir dos quais a "construção de significados" pode acontecer. Para Wilson (1999), algum tipo de incerteza resulta de imprecisas palavras e idéias - reduzir esse tipo de incerteza é construir "significados comuns".

Em outro trabalho, Wilson (2002) qualifica informação como um conceito socialmente construído, e, como parte de um processo social, informação está relacionada com a percepção humana, com a idéia de "significado". Wilson também destaca que as abordagens humanísticas geralmente vêem a realidade social como construída por meio da ação social da parte das pessoas, que levam a efeito atos que têm significado para elas.

Para Dobson (2002), nosso conhecimento da realidade é um resultado do condicionamento social, e, portanto, não pode ser entendido independentemente dos atores sociais envolvidos no processo de derivação de conhecimento. Wilson (1980) destaca que diferentes atores têm diferentes percepções do mesmo fenômeno ou situação.

Construção social e significado, portanto, tornam-se centrais para a maioria dos humanistas, em contraste com visões positivistas da natureza da realidade social, na qual fatos sociais podem ser conhecidos com certeza e na qual leis de causa e efeito podem ser descobertas e aplicadas (WILSON, 1980).
Para Allen e Wilson (2003), o significado "é transmitido, reproduzido e criado por meio de símbolos e linguagem”. De acordo com esses autores, o significado organizacional restringe a ação de seus próprios criadores. Esse tipo de construto poderia ser mais entendido com a análise das sagas organizacionais, lendas, mitos e estórias, as quais moldam a cultura organizacional.

Para Allen (2000), diferentes organizações, com diferentes culturas organizacionais, irão, inerentemente, ter visões diferentes de como um dado sistema poderia ser gerenciado, usado e desenvolvido. Dando suporte a essas diferenças, estão perspectivas organizacionais e profissionais muito diferentes (GLENDINNING, 2003). Assim, o sucesso de um ambiente informacional cooperativo depende de quão bem gerenciadas forem as diferentes culturas organizacionais.

Também os relacioamentos interpessoais freqüentemente influenciam o compartilhamento de informações, de acordo com Kolekofski (2003). Amizade e contatos pessoais influenciam fortemente a comunicação entre indivíduos. Kolekofski ainda destaca que esses fatores contribuem para o incremento do compartilhamento da informação. Auto-interesse e reciprocidade também desempenham papéis importantes no compartilhamento da informação.

Kolekofski (2003) adicionalmente menciona que a qualidade percebida da informação pode ser um importante fator para avaliar auto-interesse e reciprocidade. Se a informação comparitlhada é percebida como ruim, um temor de retribuição e culpa pode ser induzido. Entretanto, mediadores com atitudes prósociais e normas organizacionais positivas (políticas, procedimentos e cultura) podem mitigar ou superar aquele impacto negativo.

Para Castelfranchi (2002), uma ontologia existe para ser compartilhada, para ser institucionalmente padronizada ou para ser negociada entre os agentes para entendimento mútuo, intercâmbio de informações e colaboração. Adicionalmente, nenhuma comunidade, nenhuma comunicação efetiva ou cooperação é possível sem algum nível de interpretação comum do mundo e entendimento mútuo.

Uma ontologia compartilhada, porém, não nasce pronta ou como resultado de mágica; é produto de uma interpretação comum do mundo e das experiências, da interação e cooperação, e alguma forma de negociação implícita ou explícita sobre significados e como os problemas sociais de compartilhamento e de mútuo entendimento são realmente as questões subliminares 
que importam. É necessário, então, oferecer oportunidades para construir o entendimento mútuo (AUSTIN, 2002).

Para considerar o contexto do usuário, os novos ambientes informacionais cooperativos precisam ser desenvolvidos usando uma perspectiva centrada no usuário, a despeito dos diferentes ambientes organizacionais de onde provêem os usuários. Mutschke (2001) propõe uma técnica, a que chamou de "análise sociocognitiva", para lidar com a questão do significado comum em redes. Mutschke descreve essa técnica como uma representação de metaestruturas, como a estrutura social de um campo de pesquisa que usa informação bibliográfica.

Dessa forma, os ambientes informacionais cooperativos a serem desenvolvidos devem ser socialmente especificados e integrados e precisam estar envolvidos em relacionamentos sociais reais, porque têm de atuar como mediadores entre humanos (CASTELFRANCHI, 2002). Esse tipo de ambiente irá funcionar no que Mutschke (2001) chamou de "rede social". Mutschke define "rede social" como uma comunidade comportamental e social e usa essa noção para descrever relacionamentos e interações entre os atores sociais.

Para Castelfranchi (2002), a nova palavra-chave é interação: interação com um ambiente envolvente, entre vários sistemas heterogêneos distribuídos em uma rede, com usuários humanos e entre humanos por meio de computadores e sistemas.

Assim, para enfrentar esse desafio, talvez sejam necessários não apenas os novos ambientes informacionais cooperativos, mas também novas organizações. Como seria, porém, esta "nova organização" requerida pelo novo paradigma?

\section{AS NOVAS ORGANIZAÇÕES}

Existe uma profusão de termos correntemente usados para descrever a "nova organização" sugeridos pelos autores e a seguir referidos: organização ágil, organização em rede, organização virtual, organização extendida (extended enterprise), organização do conhecimento (knowledge enterprise), organização do aprendizado (learning organisation) e organização "inteligente" (smart organisation) (ALADWANI, 2002; BAKER, 2002; BRADNER, 2003; BURNETT, 2000; CAMARINHAMATOS, 2001; CARBO, 2003; CASTELFRANCHI, 2002; DEVINE, 2001; FILOS, 2001; FRENKEL, 2001; GORANSON, 2001; INKPEN, 2001; MERALI, 2002;
MOLINA, 2001; MUNDIM, 2001; RICCI, 2002; RIEMPP, 1998; SHUMAR, 2002; SILVA, 2001).

Goranson (2001) assume que organizações virtuais podem ser desenvolvidas nas seguintes situações: a) quando uma organização singular se divide em várias organizações distribuídas; b) quando o trabalho é distribuído entre várias organizações diferentes; c) quando a organização virtual é "oportunista", isto é, quando ela é desenvolvida apenas para aproveitar uma oportunidade, sem senso de permanência. Em qualquer situação, um ambiente informacional cooperativo é condição para o sucesso da organização virtual.

Essa relação entre o conceito de organização virtual e redes informacionais deriva da noção implícita de agilidade presente no conceito. Para Devine (2001), uma organização virtual é uma coleção de entidades geograficamente distribuídas e funcional e/ou culturalmente diversas, que são conectadas por meio de tecnologias da informação e comunicação e que apóiamse em relacionamentos laterais e dinâmicos para a necessária coordenação das ações.

Para Silva (2001), conceitos organizacionais como supply chain, extended enterprise e virtual enterprise sugerem idéias de distribuição, descentralização, dinamismo, autonomia e agilidade. Frenkel (2001) declara que organizações virtuais representam colaboração de grupos de organizações autônomas e preexistentes, as quais seletivamente compartilham expertise, habilidades e recursos, de forma a alcançar um produto ou serviço comum.

Para Riempp (1998), uma organização virtual é uma coalizão temporária de várias organizações legalmente independentes, com o propósito de oferecer conjuntamente um produto ou um serviço. Também para Camarinha-Matos (2001), uma organização virtual é um consórcio de organizações que compartilham habilidades e recursos. No mesmo sentido, Molina (2001) define organização virtual como redes temporárias de organizações independentes, conectadas pela tecnologia da informação, as quais compartilham competências, infra-estrutura e processos de negócio, com o propósito de atender a uma demanda específica do mercado.

Para Ricci (2002), uma organização virtual ocorre em resposta às necessidades do consumidor e é uma agregação temporária de organizações autônomas e possivelmente heterogêneas, concebida para prover flexibilidade e adaptabilidade às mudanças freqüentes que caracterizam os cenários de negócio. 
Assim, modelos e tecnologias para uma organização virtual têm de fazer face aos fortes requerimentos resultantes da necessidade de integrar e coordenar atividades distribuídas, as quais devem cooperar e se comunicar entre si, a despeito da heterogeneidade de plataformas tecnológicas e da imprevisibilidade do ambiente.

Para Mundim (2001), vários fatores direcionam a crescente cooperação entre organizações, como as rápidas transformações tecnológicas, o processo de globalização e a tendência de concentração em competências-chave (core competencies). Uma organização virtual seria baseada na integração de competências entre organizações independentes, provendo um produto ou serviço que não poderia ser oferecido em tempo e com custo adequado por nenhum dos parceiros sozinho.

Entretanto, a despeito do fato de que as organizações estão se movendo em direção à cooperação, a transição na era digital envolve muito mais que estabelecer uma infra-estrutura tecnológica e requer ainda mais que apenas a habilidade de colaborar com parceiros. O desafio inclui aprender a desenvolver projetos envolvendo diferentes times, de diferentes organizações, e algumas questões como fronteiras e integração social devem ser consideradas: como promover integração e como o trabalho cooperativo pode ter sua performance aumentada?

Nesse novo contexto, a necessidade de confiança e o nível de confiança devem ser objeto de especial atenção no desenvolvimento e gestão de parcerias. O estabelecimento de apropriados níveis de confiança e controle dependerá de fatores culturais e organizacionais (CARBO, 2003; CASTELFRANCHI, 2002).

Outras questões, como identidade organizacional, integridade e propriedade, emergem quando as organizações utilizam a tecnologia para desenvolver novos tipos de relacionamentos, compartilhando recursos e processos através de fronteiras redefinidas no cyberspace ((MERALI, 2002).

Quanto à duração dos relacionamentos, apesar de comumente o conceito de organização virtual estar associado a uma associação de curta duração (CAMARINHA-MATOS, 2001; MOLINA, 2001, RICCI, 2002; RIEMPP, 1998), alguns relacionamentos parecem não ter tempo definido para terminar, e algumas alianças estratégicas persistem além da vida útil anteriormente prevista (INKPEN, 2001).
Neste ponto, cabe destacar a diferença entre a nova abordagem colaborativa a ser desenvolvida no escopo de uma organização virtual e as iniciativas de aplicação de processos e sistemas que conectam organizações, como o intercâmbio eletrônico de dados (electronic data interchange - EDI), o vendor managed replenishment (VMR) (comumente adotado em conjunto com o just-in-time (JIT)) e o gerenciamento da cadeia de suprimento (supply chain management - SCM) (LI; WILLIAMS, 1999; RIEMPP, 1998; HILL; FERGUSON, 1989; CROOK; KUMAR, 1998; GREGOR; JOHNSTON, 1998).

Enquanto nas iniciativas citadas cada organização pode ser percebida de forma isolada, cumprindo determinada função em um processo produtivo, para efeito deste trabalho uma organização virtual é definida como uma parceria entre organizações legalmente independentes, com o propósito de conjuntamente oferecer produtos ou prover serviços aos clientes/usuários, os quais a percebem como uma organização singular. Em uma organização virtual, os parceiros compartilham informações e infraestrutura de uma maneira sinérgica, incrementando a efetividade para um nível que nenhum deles poderia alcançar sozinho.

No setor público, a questão central é como os planos governamentais deveriam ser formulados e implementados, levando em conta a coordenação interorganizacional e a gestão cooperativa da informação, não apenas entre organizações púbicas de todos os níveis de governo (federal, estadual e municipal), mas também envolvendo organizações privadas e não-governamentais. Referidos aspectos parecem ter um papel fundamental a desempenhar no sucesso dos planos governamentais, como será apresentado a seguir.

\section{PLANEJAMENTO GOVERNAMENTAL E COORDENAÇÃO INTERORGANIZACIONAL}

Para cumprir suas responsabilidades, o governo desenvolve políticas públicas que são guias para atingir certos objetivos, por meio do uso dos meios disponíveis (AWAMLEH, 1990, BAILEY, 1990). Política pública significa o escopo de atividades associadas ao governo e seus agentes - o assim chamado setor público (LEVITT, 1980).

Richards (2002) afirma que a palavra "política" é um termo geral usado para descrever a decisão formal ou plano de ação adotado por um agente, seja ele um indivíduo, organização ou governo, de forma a atingir uma meta específica. Quanto à expressão "política pública", Richards a descreve como um termo específico aplicado a uma decisão formal ou plano de ação a ser 
levado a efeito, ou que, pelo menos, envolve uma organização estatal.

Para Levitt (1980), política pública ocorre em conexão com as responsabilidades do governo em uma sociedade democrática, na qual os membros da sociedade (indivíduos e grupos) explicitamente delegam certos poderes e deveres a instituições governamentais e esperam ser afetados, positivamente, por alguns dos resultados da ação governamental.

Apesar do fato de o governo desenvolver políticas específicas para diferentes necessidades e expectativas, Levitt (1980) destaca que a separação das políticas públicas em áreas de fato distorce o quadro real. Ainda que isso seja comumente feito e facilite a análise, políticas públicas são mais acuradamente percebidas como se sobrepondo e trocando influências que interagem entre si e com outras forças. Para Levitt, tratar as políticas públicas de forma compartimentada e descrever fronteiras em torno delas é mais parte do imaginário do que da realidade, mesmo que isso seja conveniente por razões descritivas.

Para Richards (2002), a arena política tem se tornado visivelmente mais congestionada - existem mais atores envolvidos, as fronteiras entre as esferas pública e privada se tornaram menos precisas, e o comando do governo sobre o processo político precisou mudar. Richards se refere a isso como um quadro de governança:

Governança é um termo usado para destacar a natureza da mudança no processo político nas décadas recentes. Em particular, ele chama a atenção para a crescente variedade de terrenos e atores envolvidos na elaboração de políticas públicas. Assim, ele demanda que nós consideremos todos os atores e terrenos envolvidos, além do core executive, no policy making process.

O conceito de governança apresentado é importante porque o governo opera em um ambiente diversificado, fragmentado, complexo e descentralizado requerendo a atuação de verdadeiros "empreendedores políticos" (KRANE, 1990). Neste processo, a preocupação do setor público com accountability poderia promover a importação de práticas gerenciais similares àquelas existentes no setor privado (ELCOCK, 1990).

Mandell (1990) concorda que é importante aplicar ao setor público alguns conceitos do setor privado, mas destaca que os gestores públicos podem precisar desenvolver outros modelos que mais precisamente reflitam os diferentes contextos do setor público. Mandell exemplifica sua advertência com a tentativa de aplicar o conceito de gestão estratégica para a gestão no setor público.

O conceito de gestão estratégica é baseado em uma perspectiva intra-organizacional com ênfase no comportamento estratégico de um gestor dentro de sua própria organização. Gestão estratégica implica que há uma relação direta entre a formulação da estratégia e a implementação baseada no comportamento estratégico do gestor e de sua habilidade em controlar relações externas, e no setor público isso pode ser uma difícil tarefa, porque gestores públicos estão habitualmente envolvidos em um complexo sistema de redes (AGRANOFF, 1990).

Dessa forma, distinguir entre modelos de gestão estratégica de acordo com a perspectiva (intra ou interorganizacional) é crítico para o entendimento dos diferentes comportamentos requeridos em redes interorganizacionais. Para Mandell (1990), em uma rede intra-organizacional, o controle é percebido a partir de uma autoridade legitimada que flui de maneira hierárquica. Estratégias são desenvolvidas pela alta gerência da organização com o entendimento de que elas serão implementadas por membros da organização que, por sua vez, são responsáveis perante aqueles que estão no topo da cadeia hierárquica.

Porém, isso não significa que todas as estratégias são implementadas da forma que a alta gerência planejou. Assim, a ênfase da alta gerência deve ser na habilidade em controlar as ações dos membros da organização, e não apenas na autoridade legitimada que detém.

Estendendo seu argumento, Mandell (1990) afirma que, em um modelo interorganizacional, o controle não é o relacionamento dominante. Os membros de uma rede podem representar diferentes níveis de governo, não havendo, necessariamente, uma relação hierárquica entre eles.

De fato, a realidade é que diferentes níveis de governo podem agir e freqüentemente agem como unidades autônomas ou semi-autonômas, dependendo do sistema de governo. Adicionalmente, uma rede também pode incluir membros que são de um mesmo nível de governo ou que representam entidades do setor privado ou entidades sem fins lucrativos.

A conseqüência é que, ao contrário do setor privado, no qual conglomerados são controlados por uma organização "pai", cada membro de uma rede do setor público pode agir independentemente dos outros membros. Assim, a 
natureza do poder em uma rede interorganizacional é distinta daquela encontrada em relações intraorganizacionais.

Com base em uma perspectiva intraorganizacional, o sucesso na implementação das estratégias definidas pela alta gerência depende da habilidade desta em controlar as ações dos membros da organização. Em uma rede interorganizacional, isso não acontece, porque os membros dessas redes precisam de outros para cumprir as próprias metas. Assim, o poder em uma rede interorganizacional reside na capacidade de uma particular organização para influenciar a viabilidade da rede.

Dessa forma, o poder em uma rede interorganizacional no setor público precisa ser visto como diverso, não descentralizado, porque cada organização da rede tem, potencialmente, igual poder. A conseqüência é que a gerência da rede não pode ser baseada na imposição de poder, mas deve ser baseada na habilidade de efetivamente barganhar e negociar com os demais membros da rede (MANDELL, 1990).

Outra distinção entre a gestão de redes intra e interorganizacional é a diversidade de ambientes. Uma rede intra-organizacional, pela própria natureza, é baseada nas ações de um gestor específico em um particular ambiente organizacional. Gestores no setor público, porém, podem ser envolvidos em mais de uma rede ao mesmo tempo.

Estas redes podem se sobrepor e freqüentemente influenciam as ações umas das outras, sendo de primária importância a necessidade de entender que estratégias em uma rede interorganizacional não podem ser desenvolvidas baseadas nos tradicionais conceitos aplicados aos ambientes intra-organizacionais.

De acordo com Mandell (1990), as três principais características da gestão intergovernamental são resolução de problemas, redes de comunicação e comportamento estratégico. Essas características se referem à habilidade para atingir coordenação e controle, para desenvolver um padrão de contatos dentro de um sistema de redes e para gerenciar interdependências - a ênfase é a habilidade de gerenciar relações em um sistema de organizações, o que pode implicar algum tipo de "barganha" entre as organizações participantes.

O processo de barganha em redes é ressaltado por Bruijn (2002), Levitt (1980) e Younis (1990), que destacam que o processo decisório em redes organizacionais é um jogo de "toma lá dá cá" (give and take). Os vários atores em uma rede de organizações constantemente estão envolvidos em um jogo no qual os problemas de um jogador estão conectados às soluções de outro jogador.

Assim, como em uma organização virtual, o processo de planejamento no setor público envolve a participação de várias organizações e atores, e a capacidade de coordenar as teias de interesses e recursos definirá o sucesso na formulação dos planos, o que, entretanto, não assegura o êxito na implementação. É este mundo real da implementação de políticas públicas que será discutido no próximo tópico.

\section{IMPLEMENTAÇÃO DE POLÍTICAS E COORDENAÇÃO INTERORGANIZACIONAL}

De acordo com Younis (1990), tradicionalmente os estudos de políticas públicas têm sido divididos em três estágios: elaboração de políticas, implementação de políticas e avaliação de políticas. O estágio de implementação, entretanto, recebeu por longo tempo uma abordagem tipo black box, como se as decisões políticas fossem automaticamente levadas a efeito pelo sistema da forma como planejadas e gerando os resultados esperados.

Para Krane (1990), planos estratégicos permanecem meros sonhos, se não forem transformados em ações. Assim, implementar significa produzir, alcançar, levar a efeito. Mas o que está sendo implementado? "Uma política, naturalmente”, responde Younis (1990). E uma política, de acordo com Dunsire (1990), em qualquer campo, é tão boa quanto sua implementação. De fato, a legitimidade de uma política se sustenta ou falha de acordo com o sucesso ou insucesso de sua implementação (URIO, 1990).

O’Toole (1990) afirma que existem duas abordagens básicas para o estudo de implementação de políticas que envolvem diversas organizações, largamente reconhecidas pela literatura: as perspectivas top-down e bottom-up. Para O'Toole, o tipo de estrutura administrativa que melhor serve à implementação de políticas é objeto de conflito entre pesquisadores.

Para certos tipos de políticas, controle central pode resultar em um processo de implementação relativamente tranqüilo, ainda que isso impeça o produto da implementação. Teorias baseadas na perspectiva bottom-up focalizam a importância de compatibilizar a estrutura de implementação com as complexidades do problema a ser resolvido pela política, se de fato produtos e eventualmente resultados devem ser satisfatoriamente alcançados. 
Já nos casos de problemas claramente percebidos e entendidos, passíveis de serem enfrentados pelas tecnologias conhecidas, processo e produto podem ambos se beneficiar de um esquema mais próximo do top-down. A conclusão é que tanto a perspectiva top-down quanto a perspectiva bottom-up têm mérito, mas precisam ser aplicadas cuidadosamente de acordo com o ambiente e as variáveis envolvidas.

Para Levitt (1980), Morgan (1990) e Younis (1990), é difícil e talvez não desejável separar implementação do policy-making - o mais importante seria a percepção de quando a ação dos formuladores pára e quando a ação dos implementadores começa.

Assim, políticas públicas não são apenas as intenções, mas também as ações do governo (LEVITT, 1980); e os objetivos e intenções podem sempre ser expressados de formas alternativas por meio de instrumentos alternativos. Para Levitt, os instrumentos para implementação de políticas se referem a pessoas, organizações, dispositivos e serviços por meio dos quais as políticas são expressadas; e à maneira pela qual esses instrumentos são aplicados e seus efeitos monitorados constitui o conceito de enforcement.

O conceito de enforcement, para Levitt (1980), também contempla os poderes disponíveis para observar e influenciar a implementação das políticas. Richards (2002) afirma que o governo tem acesso a capacidades coercitivas, de várias formas, pelas quais pode não apenas ordenar e assegurar seu apelo à obediência, mas também à implementação de políticas por parte da burocracia governamental.

No começo dos anos 1980, entretanto, o modelo burocrático tradicional foi desafiado por uma nova forma de relações interorganizacionais, reconhecida por Gage (1990) como networks. Richards (2002) afirma que o Estado se tornou uma coleção de networks interorganizacionais feitas por atores governamentais $\mathrm{e}$ da sociedade.

Para Richards (2002), um desafio-chave para o governo é viabilizar essas networks e encontrar novas formas de cooperação. Richards também afirma que existe uma mudança de hierarquia, no sentido weberiano, para heterarchy, que está relacionada ao trabalho em rede.

De fato, o processo de implementação ocorrerá tipicamente em networks multiorganizacionais. Networks de implementação são sistemas de atores de diferentes organizações que se envolvem no cumprimento dos objetivos de uma política, um bem coletivo, muito possivelmente por diferentes razões (AGRANOFF, 1990; LEVITT, 1980; PORTER, 1990; TRAUTH, 1990; WRIGHT, 1990).

Dessa forma, quaisquer que sejam as decisões políticas e suas conseqüências, o processo de implementação é caracterizado pela ênfase em sua natureza interorganizacional, como demonstram diversos estudos sobre casos de coordenação interorganizacional na implementação de políticas públicas (SOUZA, 2004; MENDES, 2003), particularmente com relação a programas sociais (OLIVEIRA, 2003; MAYNE, 2003; VASQUEZ, 2003; COSTA B., 2004), que, por suas características de necessária descentralização operacional, requerem montagens organizacionais e administrativas complexas, o que leva a antever problemas de coordenação de difícil equacionamento, se mantidas as abordagens hierárquicas tradicionais.

\section{CONCLUSÃO}

Este trabalho destacou a importância da coordenação interorganizacional no planejamento governamental, que deveria estar apoiada em um ambiente informacional cooperativo, gerenciado por uma organização pública virtual. Este tipo de ambiente cooperativo, de acordo com Castells (1998), pode prover a eficiência dos governos, que são dependentes da capacidade de processar informação e assegurar um processo decisório compartilhado.

Choo (1998) apresenta a informação como metarrecurso, com enfoque para a importância do gerenciamento da informação para a organização "inteligente". A partir de um ciclo informacional definido em uma estrutura de rede, Choo destaca a questão da cultura do compartilhamento da informação e da colaboração como fator-chave para a competitividade das organizações.

Nesse contexto, um claro entendimento dos processos intra e interorganizacionais é importante, particularmente para desenvolver estratégias efetivas para gerir a transformação organizacional em relação a um ambiente dinâmico e interconectado, no qual opera o planejamento governamental.

Tendo que suprir muitas e complexas necessidades sociais, e com escassos e pré-alocados recursos, o processo de planejamento governamental ainda precisa lidar com organizações com culturas e políticas de pessoal distintas, orçamentos e metas separados e provavelmente uma longa tradição de independência. 


\section{Henrique Silveira}

No novo ambiente cooperativo, é necessário promover relacionamentos estáveis e mútua confiança, maximizando benefícios e minimizando riscos, ao mesmo tempo que desaparece ou é bastante reduzido o tradicional controle direto sobre os recursos requeridos pelos processos de negócio.

No setor público, essas questões parecem especialmente relevantes devido à forte tradição de independência das organizações públicas, o que pode comprometer a motivação das organizações públicas para a adoção de formas mais colaborativas de trabalho.

Aplicar a idéia de organização virtual ao processo de planejamento governamental significa que os gestores públicos precisam interagir com um ambiente informacional cooperativo, no qual a informação requerida estaria disponível independentemente da real estrutura hierárquica e de eventuais diferenças tecnológicas dos sistemas que fornecem a informação.

Entretanto, a despeito dos esforços dos governos para incrementar o uso da tecnologia da informação, os sistemas de informação governamentais muitas vezes ainda constituem imenso conjunto de ilhas, conectadas quase que apenas por pontes virtuais (links). De forma a alcançar maior transparência e efetividade da ação governamental, uma efetiva integração das organizações públicas e de seus sistemas é requerida, algo como a migração para uma imagem continente, em contraponto ao arquipélago atualmente existente.

Mais que infra-estrutura tecnológica, entretanto, será necessário para viabilizar o entendimento e a aplicação do conceito de organizações virtuais no setor público. Os trabalhos consultados mostram que, para o sucesso na construção de organizações virtuais, é imprescindível ir além de considerações estritamente técnicas.

Assim, parece fundamental para o sucesso de ambientes informacionais cooperativos a construção de relacionamentos baseados na confiança e na estruturação de processos de gestão que assegurem a efetiva participação de todos os envolvidos. Aspectos como cultura organizacional, poder e controle, fronteiras e estruturas organizacionais, confiança e gestão cooperativa da informação deverão ser observados no contexto do planejamento governamental, caso se pretenda evoluir na aplicação do conceito de organizações virtuais no setor público.

Artigo recebido em 04/11/2005 e aceito para publicação em 05/01/2006.

\section{REFERENCIAS}

AGRANOFF, Robert. Responding to human crisis: intergovernmental policy networks. In: GAGE, Robert W.; MANDELL, Myrna P. (Ed.). Strategies for managing intergovernmental policies and networks. New York: Praeger publishers, 1990. p. 57-80.

ALADWANI, A. M. An empirical examination of the role of social integration in system development projects. Information Systems Journal, v. 12 . n. 4 , Oct. 2002.

ALBERTIN, Alberto Luiz. Administração de informática: funções e fatores críticos de sucesso. São Paulo: Atlas, 1999.

ALLEN, D. K. et al. Trust, power and inter-organisational information systems: the case of the Electronic Trading Community Translease. European Journal of Information Systems, v. 10, n. 1, p. 21-41, 2000.

ALLEN, David; WILSON, Tom. Vertical trust/mistrust during information strategy formation. International Journal of Information Management, v. 23, n. 3, p. 223-237. June 2003.

ARCIERI, F. et al. Coherence maintenance in cooperative information systems: the access key warehouse approach. International Journal of Cooperative Information Systems, v. 11. n. 3-4, Sept./Dec. 2002.

AUSTIN, James E. Meeting the collaboration challenge. In: WORKBOOK by the Peter F. Drucker Foundation for Nonprofit Management. New York: [s.n.], 2002.

AWAMLEH, Nail A. Hafez. Implementation of national policies through Jordanian public enterprises. In: IMPLEMENTATION in public policy. Hants: Dartmouth Publishing Company, 1990. p. 129-138.

BAILEY, Stephen J. Implementing local public choice: the community charge. In: IMPLEMENTATION in public policy. Hants: Dartmouth Publishing Company, 1990. p. 53-64.

BAKER, D. et al. Awareness provisioning in collaboration management. International Journal of Cooperative Information Systems, v. 11. n. 3-4 Sept./Dec. 2002.

BIO, Sérgio Rodrigues. Sistemas de informação: uma visão gerencial. São Paulo: Atlas, 1996.

BRADNER, Erin. Computer mediated communication among teams: what are "teams"and how are they "virtual"?. In: LUEG, Chistopher; FISHER, Danyel (Ed). From UseNet to CoWebs: interacting with social information spaces. London: Springer-Verlag, 2003. 262 p.

BRUIJIN, Hans de. Managing performance in the public sector. London: Routledge, 2002. 129 p.

BURNETT, Gary. Information exchange in virtual communities: a typology. Information Research, v. 5, n. 4, 2000. Disponível em: <http:/ /informationr.net/ir/5-4/paper82.html.>. Acesso em: 17 nov. 2003.

CAMARINHA-MATOS, L. M.; AFSARMANESH, H.; RABELO, R. Supporting agility in virtual enterprises. In: INTERNATIONAL FEDERATION FOR INFORMATION PROCESSING; WORKING CONFERENCE ON INFRASTRUCTURES FOR VIRTUAL ORGANISATIONS, 2., 2000, Florianopolis. Proceedings... Massachusetts: Kluwer Academic Publishers, 2001. 530 p.

CARBO, J.; MOLINA, J. M.; DAVILA, J. Trust management through fuzzy reputation. International Journal of Cooperative Information Systems, v. 12. n. 1, Mar. 2003

CASTELFRANCHI, C. The social nature of information and the role of trust. International Journal of Cooperative Information Systems, v. 11. n. 3-4, Sept./Dec. 2002.

CASTELLS, Manuel. Hacia el Estado Red?. Globalizacion economica e instituciones politicas en la era de la información. In: SEMINÁRIO INTERNACIONAL SOCIEDADE E A REFORMA DO ESTADO, 1998, São Paulo. Anais...São Paulo: [S.n.], 1998. 1-16 p. 
CHALKER, Sylvia; WEINER, Edmund. The Oxford dictionary of english grammar. Oxford: Oxford University Press, 1998. Oxford Reference Online. Disponível em: <http://www.oxfordreference.com>. Acesso em: 2006.

$\mathrm{CHOO}$, Chun W. Information management for the intelligent organization. New Jersey: ASIS, 1998.

COSTA, Bruno Lazzarotti Diniz. Desigualdade educacional e coordenação intergovernamental: o caso do Fundo de Desenvolvimento do Ensino Fundamental e Valorização do Magistério em Minas Gerais. In: CONGRESO INTERNACIONAL DEL CLAD SOBRE LA REFORMA DEL ESTADO Y DE LA ADMINISTRACIÓN PÚBLICA, 9., Madrid. Anales... Madrid: [s.n.], 2004.

CROOK, Connie W.; KUMAR, Ram L. Eletronic data interchange: a multi-industry investigation using grounded theory. Information $\mathbb{E}$ Management, n. 34, p. 75-89, 1998.

DEVINE, Mary; FILOS, Erastos. Virtual teams and the organisational grapevine. In: INTERNATIONAL FEDERATION FOR INFORMATION PROCESSING; WORKING CONFERENCE ON INFRASTRUCTURES FOR VIRTUAL ORGANISATIONS, 2., 2000, Florianopolis. Proceedings... Massachusetts: Kluwer Academic Publishers, 2001. 530 p.

DOBSON, Philip J. Critical realism and information systems research: why bother with philosophy?. Information Research, v. 7, n.2, 2002. Disponível em: <http://InformationR.net/ir/7-2/paper124.html>. Acesso em: 2006.

DUNSIRE, Andrew. Implementation theory and bureaucracy. In: IMPLEMENTATION in public policy. Hants: Dartmouth Publishing Company, 1990. p. 15-30.

EIN-DOR, Philip; SEGEV, Eli. Administração de sistemas de informação. Tradução de Marina Cunha Brenner. Rio de Janeiro: Campus, 1986.

ELCOCK, Howard. Implementing management change: leading and following. In: IMPLEMENTATION in public policy. Hants: Dartmouth Publishing Company Limited, 1990. p. 65-74.

FILOS, E.; BANAHAN, E.; Will the organization disappear? the challenges of the new economy and future perspectives. In: NTERNATIONAL FEDERATION FOR INFORMATION PROCESSING; WORKING CONFERENCE ON INFRASTRUCTURES FOR VIRTUAL ORGANISATIONS, 2., 2000, Florianopolis. Proceedings... Massachusetts: Kluwer Academic Publishers, 2001. 530 p.

FRENKEL, A. et al. Supporting information access rights and visibility levels in virtual enterprises. In: INTERNATIONAL FEDERATION FOR INFORMATION PROCESSING; WORKING CONFERENCE ON INFRASTRUCTURES FOR VIRTUAL ORGANISATIONS, 2 ., 2000, Florianopolis. Proceedings... Massachusetts: Kluwer Academic Publishers, 2001. 530 p.

GAGE, Robert. W. Key intergovernmental issues and strategies: an assessment and prognosis. In: Strategies for managing intergovernmental policies and networks. New York: Praeger publishers, 1990. p. 127-150.

GLENDINNING, Caroline. Breaking down barriers: integrating health and care services for older people in England. Health Policy, v. 65, n. 2, p. 139-151, Aug. 2003.

GORANSON, H. T. Infrastructure for the advanced virtual enterprise: a report using a Brazilian-based example. In: INTERNATIONAL FEDERATION FOR INFORMATION PROCESSING; WORKING CONFERENCE ON INFRASTRUCTURES FOR VIRTUAL ORGANISATIONS, 2., 2000, Florianopolis. Proceedings... Massachusetts: Kluwer Academic Publishers, 2001. 530 p.
GREGOR, S.; JOHNSTON, R. B. Developing an understanding of interorganizational systems: arguments for multi-level analysis and structuration theory. In: EUROPEAN CONFERENCE ON INFORMATION SYSTEMS, 8., 2000, Vienna, Austria. Proceedings... Vienna, Austria: Forthcoming, 2000.

HILL, N. C.; FERGUSON, D. M. Electronic data interchange: a definition and perspective. The Journal of Electronic Data Interchange, v. $1,1989$.

INKPEN, Andrew C.; ROSS, Jerry. Why do some strategic alliances persist beyond their useful life?. California Management Review, v. 44, n. 1 fall 2001.

KOLEKOFSKI, Keith E.; HEMINGER, Alan R. Beliefs and attitudes affecting intentions to share information in an organizational setting. Information $\mathcal{E}$ Management, v. 40, n. 6, p. 521-532, July 2003.

KRANE, Dale. Devolution as an inter-governmental reform strategy. In: Strategies for managing intergovernmental policies and networks. New York: Praeger publishers, 1990. p. 107-126.

KUHN, Thomas S. A estrutura das revoluções científicas. Tradução de Beatriz Vianna Boeira e Nélson Boeira. São Paulo: Editora Perspectiva, 1982.

LEVITT, Ruth. IMPLEMENTATION in public policy. London: Croom Helm, 1980. 213 p.

LI, Feng; WILLIAMS, Howard. New collaboration between firms: the role of interorganizational systems. In: HAWAII INTERNATIONAL CONFERENCE ON SYSTEM SCIENCES, 32., Big Island, Hawaii, 1999. Proceedings... Hawaii, 1999.

MANDELL, Myrna P. Network management: strategic behaviour in the public sector. In: GAGE, Robert W. Strategies for managing intergovernmental policies and networks. New York: Praeger publishers, 1990. p. 29-54.

MAYNE, John. Results-based governance: collaborating for outcomes. In: GRAY, Andrew; JENKINS, Bill; LEENW, Frans; (Ed.). Collaboration in public services: the challenge for evaluation. New Brunswick: Transaction, 2003.

MENDES, Marcos José. A eficácia da vinculação de recursos no federalismo brasileiro: o caso do Fundef. In: FINANÇAS PÚBLICAS PRÊMIO TESOURO NACIONAL, 7., 2002. Anais... Brasília: Universidade de Brasília, 2003. p. 275-330. Coletânea de monografias.

MERALI, Y. The role of boundaries in knowledge process. European Journal of Information Systems, v. 11 n.1, Mar. 2002.

MOLINA, Arturo; FlORES, Myrna. Exploitation of business opportunities: the role of the virtual enterprise broker. In: INTERNATIONAL FEDERATION FOR INFORMATION PROCESSING; WORKING CONFERENCE ON INFRASTRUCTURES FOR VIRTUAL ORGANISATIONS, 2., 2000, Florianopolis. Proceedings... Massachusetts: Kluwer Academic Publishers, 2001. 530 p.

MORGAN, Crawford. Asbestos policy and practice in a local authority In: IMPLEMENTATION in public policy. Hants: Dartmouth Publishing Company, 1990. p. 40-52.

MUNDIM, Ana Paula Freitas; BREMER, Carlos Frederico. Design of a computer-supported cooperative environment for small and medium enterprises. In: INTERNATIONAL FEDERATION FOR INFORMATION PROCESSING; WORKING CONFERENCE ON INFRASTRUCTURES FOR VIRTUAL ORGANISATIONS, 2., 2000, Florianopolis. Proceedings... Massachusetts: Kluwer Academic Publishers, 2001. 530 p.

MUTSCHKE, Peter; HAASE, Anabel Quan. Collaboration and cognitive structures in social science research fields: towards socio- 


\section{Henrique Silveira}

cognitive analysis in information systems. Scientometrics and Kluwer Academic Publishers Dordrecht v. 52, n. 3, p. 487-502, 2001.

OLIVEIRA, Luiz Eduardo Pereira de. Alternativas para o Tribunal de Contas da União quanto à avaliação de programas públicos no Brasil. Goiânia: [s.n.], 2003.

O'TOOLE, Laurence J. Multi-organizational implementation: comparative analysis for wastewater treatment. In: GAGE, Robert W.; MANDELL, Myrna P. (Ed.). Strategies for managing intergovernmental policies and networks. New York: Praeger publishers, 1990. p. 81-104.

PORTER, Michael E. How competitive forces shape strategy. In: HAMERMESH, Richard (Org.). Harvard Business review: strategic management. New York: John Wiley, 1983. p. 35-49.

RICCI, A.; OMICINI, A.; DENTI, E. Virtual enterprises and workflow management is agent coordination issues. International Journal of Cooperative Information Systems, v.11, n. 3-4, Sept./Dec. 2002.

RICHARDS, David; SMITH, Martin J. Governance and public policy in the United Kingdom. Oxford: Oxford University Press, 2002. 312 p.

RIEMPP, Gerold. Wide area workflow management: creating partnerships for the $21^{\text {st }}$ century. London: Springer-Verlag London, 1998. 318 p.

SAVIANI, José R. O analista de negócios e da informação: o perfil moderno de um profissional que utiliza informática para alavancar os negócios empresariais. São Paulo: Atlas, 1998.

SCHULTZE, Ulrike; BOLAND, Richard J. Place, space and knowledge work: a study of outsourced computer systems administrators. Accounting Management and Information Technologies, v. 10, n. 3, p. 187 219, July 2000.

SHIMIZU, Tamio. Processamento de dados: conceitos básicos. São Paulo: Atlas, 1994.

SHUMAR, Wesley; RENNINGER, K. Ann. On conceptualizing community. In: _______._Building virtual communities: learning and change in cyberspace. Cambridge: Cambridge University Press, 2002. $381 \mathrm{p}$.

SILVA, Nuno; ROCHA, Joao V. E. Infrastructures requirements for cooperation and knowledge sharing. In: INTERNATIONAL FEDERATION FOR INFORMATION PROCESSING; WORKING CONFERENCE ON INFRASTRUCTURES FOR VIRTUAL ORGANISATIONS, 2., 2000, Florianopolis. Proceedings... Massachusetts: Kluwer Academic Publishers, 2001. 530 p.

SOUZA, Saulo Santos. Entre performance política e performance fiscal: o legado do governo Cardoso para o federalismo brasileiro. In: PRÊMIO TESOURO NACIONAL 8., 2003, Brasília. Anais... Brasília:
Ministério da Fazenda, Secretaria do Tesouro Nacional, 2004. p. 403462. Coletânea de Monografias.

SPINOSA, Luiz Marcio; QUANDT, Carlos. An IT-based framework for knowledge management in networked organisations. In: INTERNATIONAL FEDERATION FOR INFORMATION PROCESSING; WORKING CONFERENCE ON INFRASTRUCTURES FOR VIRTUAL ORGANISATIONS, 2., 2000, Florianopolis. Proceedings... Massachusetts: Kluwer Academic Publishers, 2001. 530 p.

TRAUTH, Eileen; HUNTLEY, John; PITT, Douglas. The implementation game: deregulating US telecommunications. In: IMPLEMENTATION in public policy. Hants: Dartmouth Publishing Company, 1990. p. 103-116.

URIO, Paulo; HAYOZ, Nicolas. The implementation of swiss highway policy. In: IMPLEMENTATION in public policy. Hants: Dartmouth Publishing Company, 1990. p. 89-102.

VASQUEZ, Daniel Arias. Educação e desigualdades regionais: os impactos fiscais do Fundef. In: FINANÇAS PÚBLICAS PRÊMIO TESOURO NACIONAL, 7., 2002, Brasília. Anais... Brasília: Universidade de Brasília, 2003. p. 433-494. Coletânea de monografias.

WILSON, Tom. Information science and research methods: kni nicná a informacná veda (Library and Information Science). Bratislava, Slovak Republic: Department of Library and Information Science, Comenius University, 2002.

et al. Uncertainty in information seeking. Library and Information Commission Research Report, v. 59, Dec. 1999.

Recent trends in user studies: action research and qualitative methods. Berlin: Freie Universität, Institut fûr Publizistik und Dokumentationswissenschaft, 1980 . Projekt Methodeninstrumentarium zur Benutzerforschung in Information und Dokumentation, MIB P1 11/80. Disponível em: <http:// InformationR.net/ir/paper76.html>. Acesso em: 17 nov. 2003.

WRIGHT, Deil S. Federalism, intergovernmental relations and intergovernmental management: conceptual reflections, comparisons and interpretations. In: GAGE, Robert W.; MANDELL, Myrna P. Strategies for managing intergovernmental policies and networks. New York: Praeger publishers, 1990. p. 151-180.

YOUNIS, Talib; DAVIDSON, Ian. The study of implementation. In: IMPLEMENTATION in public policy. Hants: Dartmouth Publishing Company, 1990. p. 3-14.

YOURDON, Edward. Análise estruturada moderna. Tradução de Dalton Conde de Alencar. Rio de Janeiro: Campus, 1990. 